\title{
Bimodal Optomotor Response to Plaids in Blowflies: Mechanisms of Component Selectivity and Evidence for Pattern Selectivity
}

\author{
Aman B. Saleem, ${ }^{1,2 *}$ Kit D. Longden, ${ }^{1 \star}$ Daniel A. Schwyn, ${ }^{1}$ Holger G. Krapp, ${ }^{1}$ and Simon R. Schultz ${ }^{1}$ \\ ${ }^{1}$ Department of Bioengineering, Imperial College London, London, SW7 2AZ, United Kingdom, and 'Institute of Ophthalmology, University College \\ London, London, EC1V 9EL, United Kingdom
}

\begin{abstract}
Many animals estimate their self-motion and the movement of external objects by exploiting panoramic patterns of visual motion. To probe how visual systems process compound motion patterns, superimposed visual gratings moving in different directions, plaid stimuli, have been successfully used in vertebrates. Surprisingly, nothing is known about how visually guided insects process plaids. Here, we explored in the blowfly how the well characterized yaw optomotor reflex and the activity of identified visual interneurons depend on plaid stimuli. We show that contrary to previous expectations, the yaw optomotor reflex shows a bimodal directional tuning for certain plaid stimuli. To understand the neural correlates of this behavior, we recorded the responses of a visual interneuron supporting the reflex, the $\mathrm{H} 1$ cell, which was also bimodally tuned to the plaid direction. Using a computational model, we identified the essential neural processing steps required to capture the observed response properties. These processing steps have functional parallels with mechanisms found in the primate visual system, despite different biophysical implementations. By characterizing other visual neurons supporting visually guided behaviors, we found responses that ranged from being bimodally tuned to the stimulus direction (component-selective), to responses that appear to be tuned to the direction of the global pattern (pattern-selective). Our results extend the current understanding of neural mechanisms of motion processing in insects, and indicate that the fly employs a wider range of behavioral responses to multiple motion cues than previously reported.
\end{abstract}

\section{Introduction}

Moving creates patterns of visual motion across our eyes that provide valuable information for controlling our balance, motor actions and gaze. Exploiting these patterns for behavioral control requires the nervous system to detect the direction of visual motion. The neurophysiological mechanisms of motion perception have been studied in a number of systems, including insects and primates, leading to an understanding of some common principles of local motion detection (Hassenstein and Reichardt, 1956; Adelson and Bergen, 1985). A fruitful approach has been to take a pair of drifting sinusoidal gratings and combine them additively to produce a plaid stimulus (Fig. 1a). In a breakthrough study for the identification of the neural correlates of perception (Newsome et al., 1989), the behavioral measure of plaid perception in primates correlated with the activity of a subset of neurons within visual cortical area V5/MT. Plaid stimuli have been used since to

\footnotetext{
Received Sept. 28, 2011; revised Nov. 30, 2011; accepted Dec. 6, 2011.

Author contributions: A.B.S., K.D.L., D.A.S., H.G.K., and S.R.S. designed research; A.B.S., K.D.L., and D.A.S. performed research; A.B.S., K.D.L., D.A.S., H.G.K., and S.R.S. analyzed data; A.B.S., K.D.L., D.A.S., H.G.K., and S.R.S. wrote the paper.

${ }^{*}$ A.B.S. and K.D.L. contributed equally to this study.

This work was supported by Air Force Research Laboratory Award FA8655-09-1-3022 to H.G.K., and by the Gatsby Charitable Foundation Grant GAT2830 to S.R.S. Thanks to B. Haider, M. Wicklein, and anonymous reviewers for comments on the manuscript, and to A. Borst for helpful discussions.

Correspondence should be addressed to Kit D. Longden or Aman B. Saleem, Department of Bioengineering, Imperial College London, London, SW7 2AZ, UK. E-mail: kit@imperial.ac.uk or aman.saleem@ucl.ac.uk.

DOI:10.1523/JNEUROSCI.4940-11.2012

Copyright $\odot 2012$ the authors $\quad 0270-6474 / 12 / 321634-09 \$ 15.00 / 0$
}

make significant progress in accounting for the properties of cells involved in primate motion perception (Simoncelli and Heeger, 1998; Rust et al., 2006).

At the same time, our understanding of visual motion processing benefits from studies of model systems in which individually identifiable cells contribute to visually guided behavior (Hartline et al., 1956; Bialek et al., 1991; Gabbiani et al., 2002). The blowfly has well described flight and gaze stabilization reflexes, both of which are heavily supported by a set of optic flow-processing interneurons, the lobula plate tangential cells (LPTCs), that have been extensively studied (Hausen, 1993; Borst and Haag, 2002; Krapp and Wicklein, 2008). LPTCs integrate the outputs of multiple local, directionselective elements, known as elementary motion detectors (EMDs), to form extended motion-sensitive receptive fields (Hassenstein and Reichardt, 1956; Egelhaaf and Borst, 1993; Krapp and Hengstenberg, 1996). The computations performed by EMDs are well understood mathematically, and the compound eye geometry places simplifying constraints on the spatial organization of their visual inputs (Petrowitz et al., 2000; Sanes and Zipursky, 2010).

We have investigated for the first time how a visually guided insect processes plaids. Previous studies on the integration of two-dimensional pattern motion in LPTCs have assumed that a cell's output is well described by the inner product between the stimulus direction and the cell's preferred direction (Eriksson, 1984; Reichardt and Schlögl, 1988; Borst et al., 1993), exactly as in the pattern cell model of mammalian visual motion studies (Eq. 1, Materials and Methods; Movshon et al., 1985). This assumption predicts that LPTCs and the behaviors that they control 
a

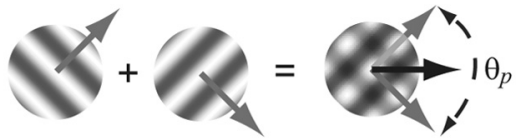

b

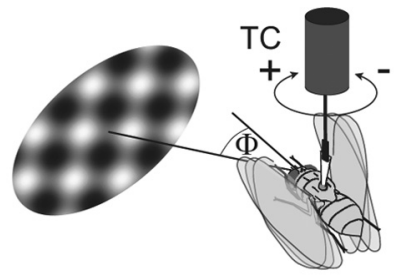

d

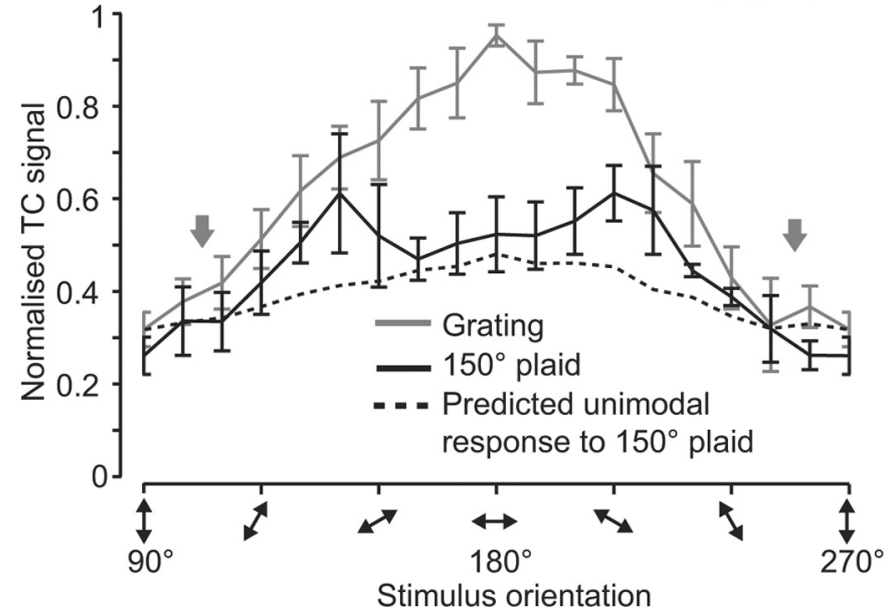

Figure 1. Bimodal optomotor response to plaid stimulus. $a, A$ plaid is formed from two half-contrast gratings, to form a full contrast pattern. Gray arrows indicate direction of motion of gratings, black arrow indicates direction of plaid motion. $\theta_{\mathrm{p}}$ denotes the plaid separation angle. $\boldsymbol{b}$, Diagram of apparatus. Positive and negative torque directions for the torque compensator (TC) are shown. The monitor is centered at azimuth angle, $\Phi$, of $30^{\circ}$. c, Temporal frequency $(F)$ of plaid stimulus, and example torque compensator trace. $\boldsymbol{d}$, Responses to a single grating (gray), $150^{\circ}$ plaid (black), and predicted pattern cell response (dashed). Mean \pm SE shown $(N=5)$. Gray arrows indicate the angles at which the plaid components are moving horizontally, $105^{\circ}$ and $255^{\circ}$.

should have a unimodal tuning to plaids (Fig. 1d). We show that, in fact, the blowfly optomotor reflex can have a bimodal tuning to plaids, as can the activity of a supporting LPTC, the $\mathrm{H} 1$ cell. To explain these results, we show it is necessary to model properties of EMDs whose functional consequences have not previously been fully appreciated (Zanker, 1990; Borst, 2004). We also show that the properties of other LPTCs involved in visually guided behavior range from bimodal responses to plaid components, to unimodal responses that appear to be selective to the direction of pattern motion.

\section{Materials and Methods}

\section{Optomotor experiments}

Male blowflies, Calliphora vicina, aged 2-4 weeks, were used. A mixture of beeswax and rosin was used to wax a small strip of cardboard to the fly's thorax, by which the fly was held in a torque compensator (Götz, 1964). The fly rested for $\geq 1 \mathrm{~h}$ with food and water before experiments.

Each plaid stimulus was generated by the superimposition of two halfcontrast gratings moving in different directions (Fig. 1a). The directions of the underlying gratings were rotated to form a plaid stimulus moving with a specific plaid direction. The half-contrast gratings had a spatial wavelength of $11.5^{\circ}$ and a temporal frequency of 2 cycles/s. The stimuli were displayed with a Michelson contrast of $60 \%$ in a circular aperture of diameter $60^{\circ}$ centered at $30^{\circ}$ azimuth (az.) $0^{\circ}$ elevation (el.). The stimuli were displayed on an Iiyama 19 inch CRT monitor (Vision Master Pro 454 monitor) at $200 \mathrm{~Hz}$, and generated using Psychophysics Toolbox (Brainard, 1997). Stimuli were displayed in a random order of orientations between $90^{\circ}$ and $270^{\circ}$, in $10^{\circ}$ steps, with 20 trials at every orientation. The stimulus was presented for 5 cycles in a trial. In a cycle, the pattern moved in the positive direction for $1 \mathrm{~s}$, then in the opposite direction for $1 \mathrm{~s}$. An isoluminant screen was presented before and after trials for $\geq 2 \mathrm{~min}$, while the fly rested and fed.

The yaw torque was measured using a torque compensator (MPI), sampled at $5 \mathrm{kHz}$ with a NI USB-6251 data acquisition card (National Instruments Corp.). We calculated the mean response to each stimulus cycle by pooling responses to all cycles, across all trials. The response to the first stimulus cycle was excluded because the responses at the start of flight were highly variable. To determine the response amplitude and phase, we fit a $0.5 \mathrm{~Hz}$ sine wave to the mean cycle response using the NelderMead algorithm (Nelder and Mead, 1965). To correct for interindividual variations in yaw torques generated, the directional tuning curves of each fly were normalized by the mean response to the half-contrast grating stimuli.

\section{Electrophysiology}

Female flies aged $4-11 \mathrm{~d}$, were used. The animal preparation and extracellular recording equipment was as described in Longden and Krapp (2009). The H1, V1 and V2 cells were identified by their motion preferences throughout the visual field (Hausen, 1984; Krapp et al., 2001; Longden and Krapp, 2009). The temperature was $23.0-24.4^{\circ} \mathrm{C}$.

Plaid stimuli were displayed in a circular aperture of diameter $24^{\circ}$, centered at $-30^{\circ}$ az. $0^{\circ}$ el. for the $\mathrm{H} 1$ and $\mathrm{V} 1$ cells, and at $105^{\circ} \mathrm{az}$. and $0^{\circ}$ el. for the V2 cell. The Michelson contrast of plaids and gratings was $20 \%$ for the $\mathrm{H} 1$ cell, $30 \%$ for the V2 cell, and 30\% (see Fig. 5) or $20 \%$ for the V1 cell. Stimuli were displayed as for the optomotor experiments, except where stated. Stimuli were randomly ordered, with directions of $0-360^{\circ}$, in steps of $15^{\circ}$, with 25 trials per direction. In a trial, an isoluminant screen was displayed for 250 $\mathrm{ms}$ before and after the stimulus, which was displayed for $500 \mathrm{~ms}$. The response was calculated as the mean spike rate during the $500 \mathrm{~ms}$ stimulus. The spontaneous spike rate was calculated over the remaining 500 ms of every trial.

\section{Pattern/component classification}

The partial correlations and $z$-scores of the response to the component and pattern cell predictions were calculated as described by Smith et al. (2005). The pattern cell response, $r_{\text {pat }}(\theta)$, to the stimulus oriented in direction $\theta$, was calculated as

$$
r_{\text {pat }}(\theta)=\left(r_{\text {grat }}(\theta)-r_{\text {spont }}\right)\left[\frac{1}{2} \cos \left(\theta+\frac{\theta_{\mathrm{p}}}{2}\right)+\frac{1}{2} \cos \left(\theta-\frac{\theta_{\mathrm{p}}}{2}\right)\right]+r_{\text {spont }},
$$

where $r_{\text {grat }}(\theta)$ is the response to the grating oriented in the same direction, $r_{\text {spont }}$ is the spontaneous spike rate, and $\theta_{\mathrm{p}}$ is the plaid separation angle. The component cell response, $r_{\text {comp }}(\theta)$, was calculated as

$$
r_{\text {comp }}(\theta)=\frac{1}{2}\left[r_{\text {grat }}\left(\theta+\frac{\theta_{\mathrm{p}}}{2}\right)+r_{\text {grat }}\left(\theta-\frac{\theta_{\mathrm{p}}}{2}\right)\right] .
$$

The partial correlation of the response to the pattern prediction, $\Omega_{\text {pat }}$, was calculated from the correlation of the neuron's response to the pattern cell and component cell predictions, $\rho_{\text {pat }}$ and $\rho_{\text {comp }}$, respectively:

$$
\Omega_{\mathrm{pat}}=\frac{\left(\rho_{\mathrm{pat}}-\rho_{\mathrm{comp}} \rho_{\mathrm{pc}}\right)}{\sqrt{\left(1-\rho_{\mathrm{comp}}^{2}\right)\left(1-\rho_{\mathrm{pc}}^{2}\right)}},
$$


where $\rho_{\mathrm{pc}}$ is the correlation between the pattern and component response (Movshon et al., 1985). The partial correlation of the response to the component prediction, $\Omega_{\text {comp }}$, was similarly calculated. The $z$-score was then calculated using the Fisher $r$-to- $Z$ transform to generate significance values,

$$
Z_{\mathrm{p}}=\frac{\frac{1}{2} \log \left(\frac{1+\Omega_{\mathrm{pat}}}{1-\Omega_{\mathrm{pat}}}\right)}{\sqrt{\frac{1}{d f}}},
$$

where $d f$ is the degrees of freedom and is equal to the number of points in the tuning curve minus 3 (Movshon et al., 2003; Smith et al., 2005). The significance value, $p$, of $Z_{\mathrm{C}}$ exceeding $Z_{\mathrm{P}}$ by 1.28 is $p=0.1$.

\section{Computational model of the $\mathrm{H} 1 \mathrm{cell}$}

We modeled the processing of directional motion stimuli by the $\mathrm{H} 1$ cell as a cascade of processing stages. Our "H1 cascade model" has four stages.

(1) EMD subunit directional tuning function. The EMD subunits' directional tuning functions were modeled as a circular Gaussian, von Mises function. The response of the tuning function $c_{n}$ of the $n$th EMD subunit, in direction $\theta$, was calculated as:

$$
c_{n}(\theta)=\frac{1}{\sum_{j} \exp \left[\kappa \cos \left(\theta_{j}-\theta_{n}\right)\right]} \exp \left[\kappa \cos \left(\theta_{j}-\theta_{n}\right)\right],
$$

where $\theta_{n}$ was the preferred direction of the subunit, $j$ indexed the stimulus directions, and $\kappa$ was a free parameter for the width of tuning of all the EMD subunits.

(2) EMD subunit output function. The response of the $n$th EMD subunit, $v_{n}$, was calculated as a static sigmoid nonlinearity of $c_{n}$ :

$$
v_{\mathrm{n}}=\frac{1}{1+\exp \left[\lambda\left(c_{n}-\epsilon\right)\right]} .
$$

where $\lambda$ and $\varepsilon$ were free parameters.

(3) EMD subunit weights. The outputs of the EMD subunits were integrated with weights, $w$, to generate an input current, $u$, to the $\mathrm{H} 1$ cell:

$$
u=\sum_{n} w_{n} v_{n}
$$

where $n$ indexes the EMD subunit directions.

(4) H1 output function. The predicted output firing rate of the $\mathrm{H} 1$ neuron, $\bar{r}$, was calculated as a static exponential nonlinearity,

$$
\tilde{r}=\omega \exp (\eta u)
$$

where $\omega$ and $\eta$ were free parameters.

To identify how the non-cosine direction tuning function and the nonlinearities of the model contributed to capturing features of the data, we first created a "simplified H1 cascade model," in which we replaced the direction tuning function by a cosine function and the two nonlinear stages with linear functions. Specifically, the EMD subunit output function was the identity, $\left(v_{n}=c_{n}\right)$, and the H1 output function was the identity, $(\tilde{r}=u)$. We then added non-cosine direction tuning and the nonlinearities one-by-one and compared the performance of the model, with and without each component.

\section{Analysis of prediction accuracy}

We used two measures to estimate how well any of the models predicted the neuronal response. The first was the root mean squared error between the predicted and measured response,

$$
R M S=\left(\left\langle\left\langle(r-\tilde{r})^{2}\right\rangle_{\text {stim }}\right\rangle_{t r i a l s}\right)^{1 / 2},
$$

where $r$ is the measured response of the cell, $\tilde{r}$ is the predicted response, and angular brackets denote averaging over stimuli (stim) and trials (tri- als). As the measured response is inherently noisy, we also calculated the fraction of explainable variance $\beta$ as (Machens et al., 2004):

$$
\beta=\frac{\sigma_{r}^{2}-\sigma_{e}^{2}}{\sigma_{r}^{2}-\sigma_{n}^{2}}
$$

where $\sigma_{r}^{2}$ is the variance in the response power,

$$
\sigma_{r}^{2}=\left\langle\left\langle r^{2}\right\rangle_{\text {stim }}\right\rangle_{t r i a l s}
$$

$\sigma_{e}^{2}$ is the square of the RMS error, and $\sigma_{n}^{2}$ is the residual noise power, calculated as:

$$
\sigma_{n}^{2}=\frac{n}{n-1}\left[\left\langle\left\langle r^{2}\right\rangle_{\text {stim }}\right\rangle_{\text {trials }}-\left\langle\langle r\rangle_{\text {trials }}^{2}\right\rangle_{\text {stim }}\right]
$$

The mean and SDs of the model performances were calculated by fitting the models to the data multiple times, and calculating the mean and SDs of the model performances. The number of times the model was fit, $N_{\text {fits }}$, was 150 for the $\mathrm{H} 1$ cell models, and 175 for the V2 cell models.

\section{Model fitting}

The electrophysiology data from each cell type was pooled. There were 5 plaid separation angles, each presented in 24 directions, resulting in 120 different stimuli. Each time the model was fit, 100 stimuli were randomly chosen, and all the responses to these stimuli were allocated to the training set. The responses to the remaining 20 stimuli formed the test set. Thus the model was fit to $83 \%$ of all the data for each cell type, and tested on the remaining $17 \%$. For the $\mathrm{H} 1$ cells, there were 150 trials for each stimulus, and for the V2 cells there were 175 trials per stimulus.

We fit the model to the response to plaid stimuli and not the grating stimuli, as the grating and the plaid stimuli had the same Michelson contrast and mean luminance values, and therefore different root mean square contrast (RMS contrast, the SD of the pixel luminance). For plaid separation angles between $30^{\circ}$ and $150^{\circ}$, the calculated RMS contrast of our stimuli is nearly constant $\left(30^{\circ}: 4.8 \%, 60^{\circ}: 5.0 \%, 90^{\circ}: 5.0 \%, 120^{\circ}\right.$ : $\left.5.0 \%, 150^{\circ}: 5.2 \%\right)$, but for the grating it is much larger (7.1\%). EMDs compare the luminance of small patches of the visual stimulus, corresponding to the acceptance angles of the ommatidia, so the grating is likely to be an effectively higher contrast stimulus than the plaids. To capture the responses to the full grating, the model would need to include contrast gain control mechanisms (Harris et al., 2000; Rust et al., 2006).

A nested fitting procedure was used to fit the possible models in five steps: (1) the free parameters $\kappa, \lambda$, and $\varepsilon$ were fixed. (2) $v_{n}$ was calculated for all possible stimuli. (3) Assuming $r=u$, the optimal weights $w_{n}$ for the these free parameters were calculated by ridge regression, using a regularization parameter of $10^{-2}$ (Hastie et al., 2001). (4) The parameters of the $\mathrm{H} 1$ output nonlinearity, $\omega$ and $\eta$ were fit using linear regression. (5) The overall training error of this fit, Error ${ }_{\text {train }}$, was calculated as the mean squared error:

$$
\text { Error }_{\text {train }}=\sqrt{\sum_{k_{\text {train }}}(r-\tilde{r})}
$$

where $k_{\text {train }}$ indexes the training data, $r$ is the response of the $\mathrm{H} 1$ cell, and $\tilde{r}$ the predicted response of the model. We iterated through steps $1-5$ to find the free parameters $\kappa, \lambda$, and $\varepsilon$ that minimize Error $_{\text {train }}$ using the fminsearch function of MATLAB (The MathWorks Inc.).

To estimate the goodness of fit, the test error, Error test $_{\text {was calculated }}$ over the test data, indexed by $k_{\text {test }}$ :

$$
\text { Error }_{\text {test }}=\sqrt{\sum_{k_{\text {test }}}(r-\tilde{r})}
$$

The simplified $\mathrm{H} 1$ cascade models were optimized on a reduced set of free parameters. We avoided overfitting when adding the non-cosine direction tuning and nonlinearities to the simplified cascade model by assessing the model performance using separate testing and training data, which penalizes overfitting. We also judged the success of the model by its ability to capture features of the data, in addition to the overall fit. 
a

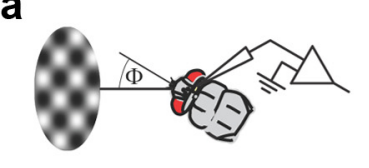

b

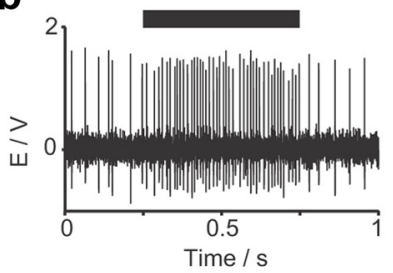

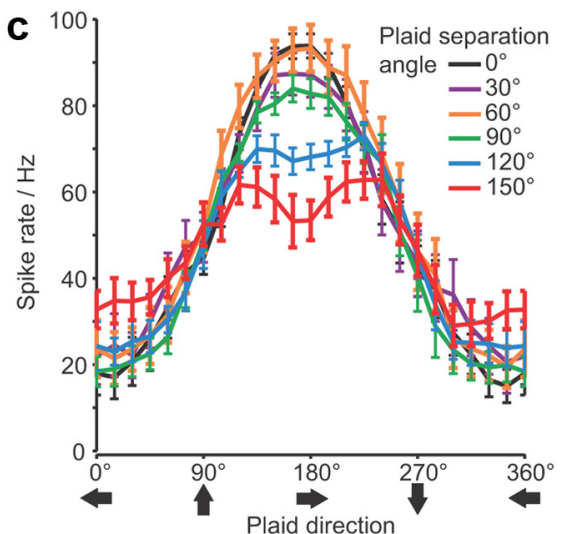
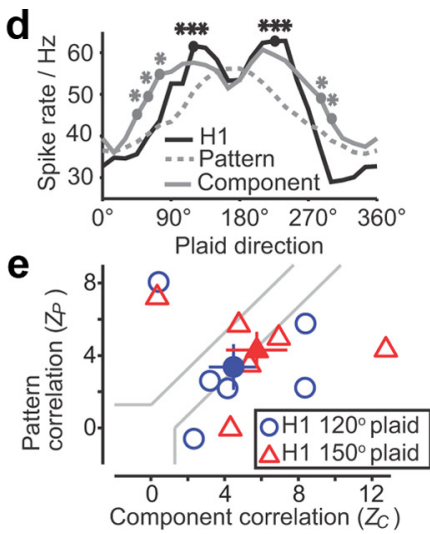

Figure 2. Component selectivity of the $\mathrm{H} 1$ cell to plaids. $\boldsymbol{a}$, Diagram of electrophysiology setup. $\boldsymbol{b}$, Example $\mathrm{H} 1$ cell response to a $60^{\circ}$ plaid. Black bar indicates stimulus period. $\boldsymbol{c}$, Mean $\mathrm{H} 1$ responses to plaids. Mean \pm SE shown $(N=6)$. $\boldsymbol{d}$, Comparison between the $\mathrm{H} 1$ response to the $150^{\circ}$ plaid, and pattern and component cell predictions. Black asterisks indicate significant differences between peak $\mathrm{H} 1$ responses, and pattern cell predictions ( $p<0.005$, paired $t$ test, $N=6$ ). Gray asterisks indicate significant differences between $\mathrm{H} 1$ responses and component cell predictions ( $p<0.05$, paired $t$ test, $N=6$ ). $e$, Normalized correlation coefficients between $\mathrm{H} 1$ responses and pattern cell predictions, $Z_{\mathrm{p}}$, and component cell predictions, $Z_{c}$, for $120^{\circ}$ plaids (blue circles) and $150^{\circ}$ plaids (red triangles). Symbols with error bars indicate the mean \pm SE. Gray lines denote statistically significant $(p=0.10)$ bias to pattern or component cell correlation (Smith et al., 2005).

\section{Results}

\section{Optomotor response to plaid stimuli}

We measured the yaw torque responses of tethered flies in flight to motion stimuli moving back and forth along different orientations (Fig. 1b). The orientation tuning to a single grating was unimodal, peaking at $180^{\circ}$, and consistent with the expected optomotor response to single gratings (Hausen and Wehrhahn, 1983) (Fig. $1 c, d$ ). The orientation tuning to the plaid, however, had a bimodal dependence on the stimulus, with peaks at $140^{\circ}$ and $210^{\circ}$ (Fig. $1 d$ ). The bimodal tuning was not well described by the tuning predicted by the inner product between the stimulus direction and the preferred direction of the fly's response to the single grating (Fig. 1d). To understand how this behavior could be generated, we recorded the responses of a neuron involved in the yaw optomotor reflex.

\section{Component motion selectivity of $\mathrm{H} 1$ cell: neural correlate of yaw optomotor response}

The H1 cell is part of a network of LPTCs sensitive to horizontal optic flow that mediates the optomotor yaw response (Hausen and Wehrhahn, 1989, 1990; Haag and Borst, 2001). Its mean spike rate is correlated to the yaw torque generated in response to moving gratings (Eckert, 1980). The tuning of the $\mathrm{H} 1$ cell to the direction of a $150^{\circ}$ plaid was bimodal, consistent with the bimodal optomotor yaw response to a $150^{\circ}$ plaid (Fig. 2). The results were not well described by the unimodal tuning predicted by the pattern cell model, with significant differences to the $\mathrm{H} 1$ response peaks at $120^{\circ}$ and $225^{\circ}$ (Fig. $2 d ; p<0.002$, paired $t$ test, $N=6$, with Bonferroni correction, $\left.N_{\text {tests }}=24\right)$.

We next compared the tuning of the $\mathrm{H} 1$ cell to the predictions of the "component cell" model, because this model is widely used to classify and understand the neural responses to plaids in vertebrate systems (Movshon et al., 2003). In the component cell model, each component of the plaid is processed independently, and the cell's response is the sum of these operations (Eq. 2, Materials and Methods) (Adelson and Movshon, 1982). The response to $150^{\circ}$ plaids was better fit by the component cell model than the predicted unimodal tuning (Fig. $2 d ; R^{2}=0.72$ vs 0.66 ), and the correlation was significantly higher for 4 of the 6 cells (Fig. $2 e ; p<0.10$; mean $Z_{\mathrm{C}}-$ mean $Z_{\mathrm{P}}=1.42, N=6$ ). The component selectivity of the $\mathrm{H} 1$ cell responses to the $150^{\circ}$ plaid was present within the $50 \mathrm{~ms}$ after the stimulus onset for 5 of the six cells $\left(p<0.10\right.$; mean $Z_{\mathrm{C}}-$ mean $\left.Z_{\mathrm{P}}=3.22, N=6\right)$.

Two observations indicated processing steps not captured by the component cell model. First, the peak responses to the $60^{\circ}$ plaid were greater than for the $30^{\circ}$ plaid (Fig. $2 c ; p<0.02$, paired $t$ test, $N=6$ ). Second, the directional tuning to the $150^{\circ}$ plaid was narrower than predicted by the component cell model (Fig. 2d). This second factor limited the magnitude of the component selectivity correlation score, $Z_{\mathrm{C}}-Z_{\mathrm{P}}$, despite the qualitative good fit of the bimodal component predictions. Across all cells, $Z_{\mathrm{C}}-$ $Z_{\mathrm{P}}$ was correlated for the $120^{\circ}$ and $150^{\circ}$ plaids (Pearson's correlation coefficient, $\rho=0.69$ ). One of the H1 cells showed clear pattern selectivity for the $120^{\circ}$ and $150^{\circ}$ plaids (Fig. $2 e$ ), despite having bimodal responses to these plaids. This cell had a more variable response to the grating, with a mean $\mathrm{SD}$ of $21.8 \mathrm{~Hz}$, compared with $14.0 \mathrm{~Hz}$ for the other cells, which skewed the pattern and component predictions for this cell.

\section{Model of the $\mathrm{H} 1$ cell response}

To understand the computational mechanisms underlying the bimodal directional tuning of LPTCs to plaids, we modeled the tuning of the $\mathrm{H} 1$ cell (Fig. 3). First we describe our $\mathrm{H} 1$ cascade model that includes both the EMD layer as well as the $\mathrm{H} 1$ cell. We then explain the contributions of the $\mathrm{H} 1$ cascade model components.

The initial layer of our $\mathrm{H} 1$ cascade model consists of $12 \mathrm{EMD}$ subunits, corresponding to interactions between pairs of nearest and next-nearest ommatidia (Fig. $3 a$ ). In the light-adapted fly, the optomotor responses can be well explained by considering only these EMDs (Buchner, 1976, 1984). Individual EMD subunits were modeled using a set of directional tuning functions (Fig. $3 b$ ), whose output was processed by a saturating nonlinearity (Fig. 3c). The EMD subunit responses were integrated as a weighted sum (Fig. $3 d$ ), and an exponential nonlinearity converted this input into a spike rate (Fig. $3 e$ ). In total, the $\mathrm{H} 1$ cascade model has 5 parameters specifying the nonlinear functions and non-cosine direction tuning, and 12 parameters specifying the EMD subunit weights. The parameters were fit to a fraction of the data using a nested fitting procedure, and were validated on the remaining data.

The $\mathrm{H} 1$ cascade model was able to capture the essential features of the experimental data (Fig. $3 f$ ): the directional tuning of 


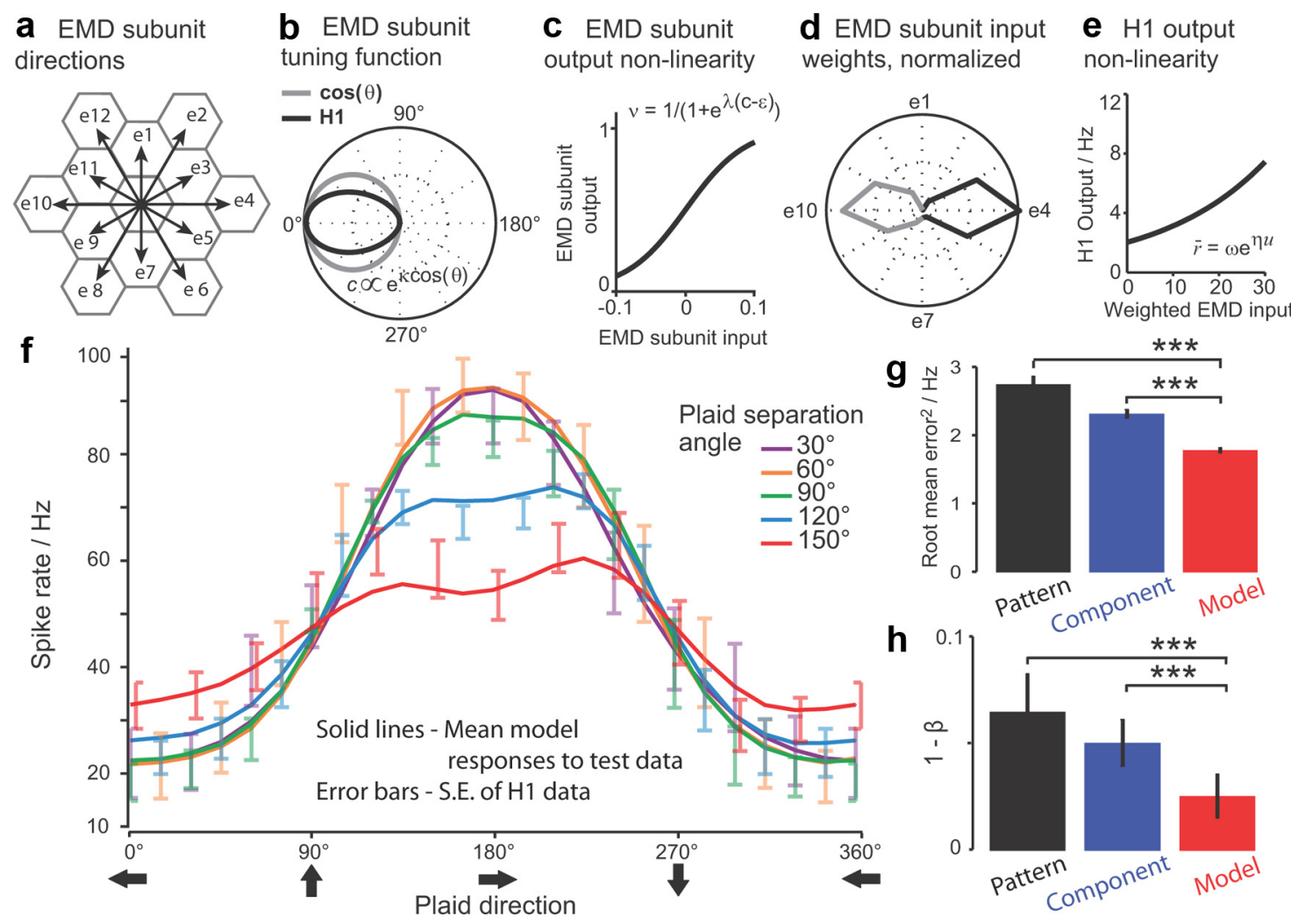

Figure 3. H1 cascade model. $\boldsymbol{a}-\boldsymbol{e}, \mathrm{H} 1$ cascade model parameters, fitted values and equations. $\boldsymbol{a}$, The 12 directions of EMD subunits (black arrows). $\boldsymbol{b}$, EMD subunit directional tuning function. The fitted function (black) is shown with a cosine for comparison (gray). One parameter, $\kappa$, determines the directional tuning. $c$, EMD subunit output nonlinearity. Two parameters, $\lambda$ and $\varepsilon$, determinethe sigmoid function. $\boldsymbol{d}$, EMD subunit weight distribution. Excitatory (black) and inhibitory (gray) weights are shown.e, H1 outputnonlinearity. Two parameters, $\omega$ and $\eta$, determine the H1 output to the weighted EMD input.f, Mean responses of $\mathrm{H} 1$ cascade model to the test data (solid lines). Error bars show the variability (SE) of the $\mathrm{H} 1$ cell recordings, to demonstrate the accuracy of the $\mathrm{H} 1$ cascade model fit. $\boldsymbol{g}$, Root mean squared errors between $\mathrm{H} 1$ cascade model responses and predictions of the pattern and component models. $\boldsymbol{h}$, Fraction of explainable variance, $\beta$, accounted for by the models. $\boldsymbol{g}, \boldsymbol{h}$, Mean $\pm \mathrm{SE}$ shown $\left(N_{\text {fits }}=150\right)$.

the cell obtained with the $150^{\circ}$ plaid was bimodal, narrower than the component prediction, and the peak response to the $60^{\circ}$ plaid was greater than that for the $30^{\circ}$ plaid $(p<0.01,2$-sample $t$ test; $\left.N_{\text {fits }}=150\right)$. Across all plaid directions, the $\mathrm{H} 1$ cascade model fit the data more accurately than the pattern and component cell models (Fig. $3 g ; p<0.001,2$-sample $t$ test; $N_{\text {fits }}=150$ ). We also used the fraction of explainable variance, $\beta$, to test the goodness of fit of the $\mathrm{H} 1$ cascade model. This measure takes into account the response variability across trials: a value of 1 identifies the best fit that can be expected from a model given this intrinsic variability. The $\mathrm{H} 1$ cascade model accounted for a significantly greater fraction of explainable variance than the pattern and component cell models (Fig. 3h; $p<0.001,2$-sample $t$ test; $N_{\text {fits }}=150$ ).

\section{Contributions of model components: mechanisms of component selectivity}

To understand how the non-cosine direction tuning and nonlinearities of the $\mathrm{H} 1$ cascade model allowed us to capture features of the $\mathrm{H} 1$ cell responses to plaids, we started with a simplified $\mathrm{H} 1$ cascade model, that was just a weighted sum of cosine-tuned EMD subunit outputs. We then added the non-cosine direction tuning function and the two nonlinearities one-by-one, and compared the performance of the model in capturing features of the data, with and without each component (Fig. 4).

To generate the bimodal tuning, it was necessary to include an EMD subunit directional tuning function that was narrower than a cosine (Fig. $4 a$ ). Only then was the optimal tuning narrower than a cosine, in agreement with the narrow, bimodal tuning of the $\mathrm{H} 1$ cell to the $150^{\circ}$ plaid (Fig. $4 a 5$ ). With the non-cosine directional tuning function, the $\mathrm{H} 1$ cascade model accounted for substantially more of the explainable variance (Fig. $4 a 1 ; \beta$ from 0.93 to $0.94 ; p<0.001 ; 2$-sample $t$ test; $N_{\text {fits }}=150$ ).

The addition of the sigmoidal nonlinearity applied to the output of each EMD subunit allowed the $\mathrm{H} 1$ cascade model to capture the greater peak response of the cell to a $60^{\circ}$ plaid compared with a $30^{\circ}$ plaid (Fig. $4 b 2,3$ ). The nonlinearity suppressed the peak responses to each component grating, and effectively boosted the response to each component grating in the anti-preferred direction. It also increased the peak responses to the $120^{\circ}$ and $150^{\circ}$ plaids to a near perfect match with the cellular responses (Fig. 4b4,5), which improved the overall fit (Fig. $4 b 1 ; \beta$ from 0.94 to $0.96 ; p<0.001$; 2-sample $t$ test).

Including an exponential $\mathrm{H} 1$ output nonlinearity resulted in a modest increase of the fit (Fig. $4 c 1 ; \beta$ from 0.964 to $0.975 ; p<0.01$; 2 -sample $t$ test). The nonlinearity amplified the difference in the peak responses to the $30^{\circ}$ and $60^{\circ}$ plaids (Fig. $4 c 2,3$ ), and improved the fit of the $\mathrm{H} 1$ cascade model in the nonpreferred directions, where the cell is inhibited (Fig. 4c2-5). However, the H1 output nonlinearity reduced the fit of the $150^{\circ}$ plaid, leading to an almost unchanged fit of the $\mathrm{H} 1$ cascade model overall (Fig. 4c5).

\section{Motion selectivity of the V1 and V2 LPTCs: evidence for pattern selectivity}

To understand whether bimodal directional tuning to plaids was a general property of LPTCs, we also recorded from two other spiking LPTCs, the V1 and V2 cells. The directional tuning of the V2 cell to plaids was qualitatively similar to that of the $\mathrm{H} 1$ cell (Fig. 5a), in particular with respect to the bimodal response to the 

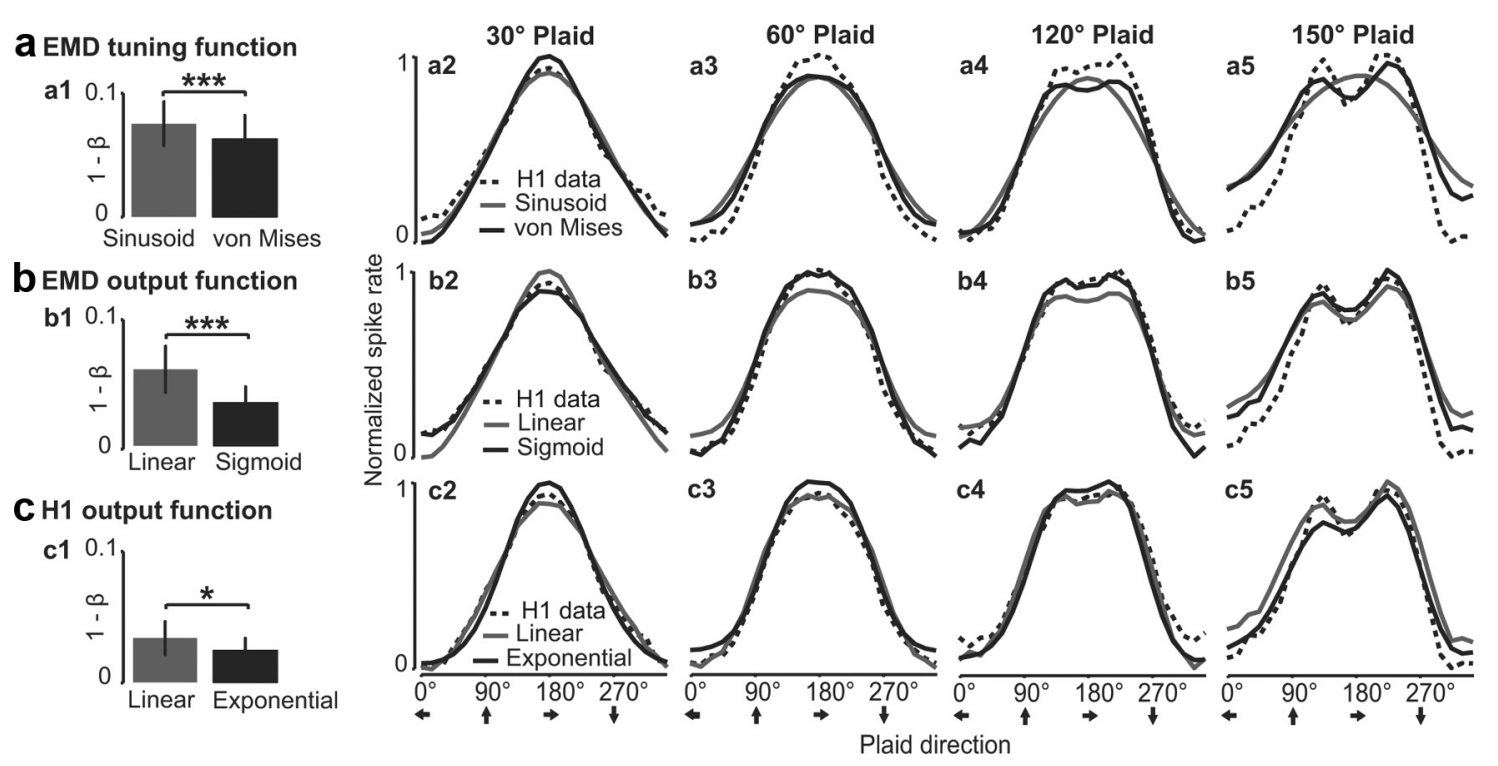

Figure 4. Contributions of $\mathrm{H} 1$ cascade model components. In every row, a component of the $\mathrm{H} 1$ cascade model is added to the simplified $\mathrm{H} 1$ cascade model, and the performance with and without the component is shown. a1, Fraction of explainable variance, $\beta$, accounted for by the simplified model when the directional tuning is a sinusoid (gray), or the nonlinear von Mises function (black). Mean \pm SD across multiple fits $\left(N_{\text {fits }}=150\right)$. a2, Fit to the $30^{\circ}$ plaid responses of the $\mathrm{H} 1$ cell (dashed) of the simplified model with the sinusoidal (gray), or von Mises (black) EMD subunit directional tuning. $\boldsymbol{a} 3-5$, As for $\boldsymbol{a} 2$, for $60^{\circ}, 30^{\circ}, 120^{\circ}$, and $150^{\circ}$ plaids. $\boldsymbol{b}$, As for $\boldsymbol{a}$, but adding the sigmoid EMD subunit output nonlinearity (Sigmoid). $\boldsymbol{c}$, As for $\boldsymbol{b}$, but adding the exponential H1 output nonlinearity (Exponential).
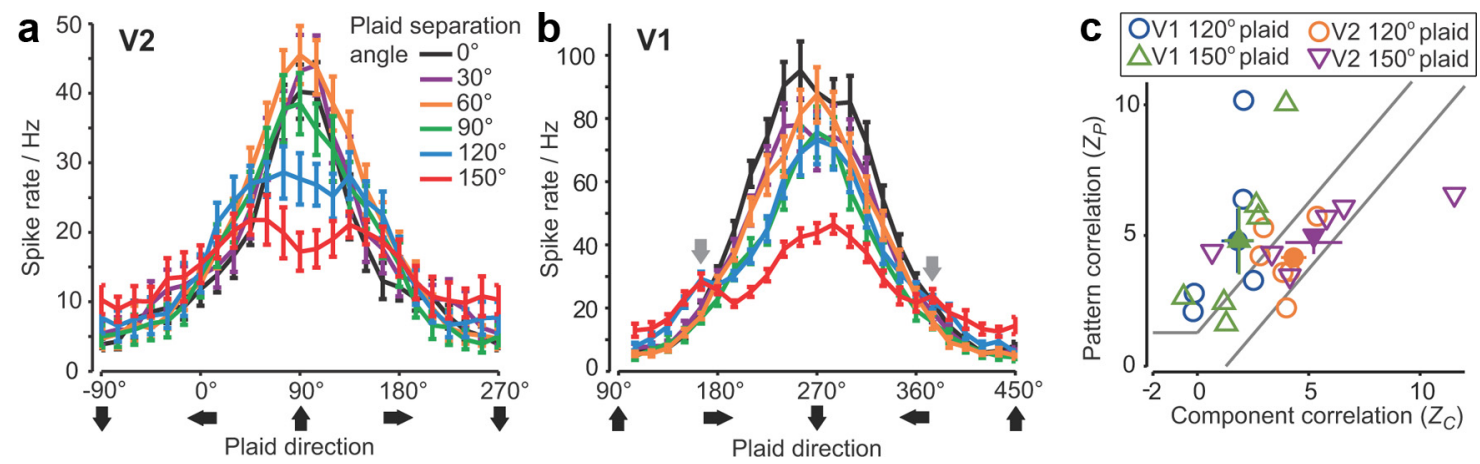

Figure 5. Pattern selectivity of $\mathrm{V} 1$ cell and response of $\mathrm{V} 2$ cell. $\boldsymbol{a}, \boldsymbol{b}$, Responses of $\mathrm{V} 2(\boldsymbol{a})$ and $\mathrm{V} 1$ cells $(\boldsymbol{b})$ to plaids. Gray arrows in $\boldsymbol{b}$ indicate secondary peaks in the response to the $150^{\circ}$ plaid. Mean \pm SE shown. V2, $N=7 ; \mathrm{V1}, N=6$. c, Normalized correlation coefficients, as in Figure 2e, for the V2 cells (orange circles and purple triangles) and V1 cells (blue circles and green triangles).

$150^{\circ}$ plaid. However, the V2 response to the $150^{\circ}$ plaid was narrower than the component cell prediction, such that the V2 responses could not be classified as pattern- or componentselective by their correlations with the pattern and component cell models (Fig. $5 c$ ).

A V2 cascade model, with a structure identical to the $\mathrm{H} 1$ cascade model, was able to fit the V2 cell data well (Fig. 6; $\beta=0.98$ for V2, $\left.N_{\text {fits }}=175\right)$. In particular, the V2 cascade model generated a peak output to the $60^{\circ}$ plaid that was greater than for the $30^{\circ}$ plaid, and a bimodal directional tuning to the $150^{\circ}$ plaid (Fig. $6 f$ ). The V2 cascade model produced a narrower EMD subunit tuning function than the $\mathrm{H} 1$ cascade model (Fig. $6 b$ ). As the interommatidial angle is greater in the visual field where the V2 cell was stimulated, compared with the region used for the $\mathrm{H} 1$ cell (Petrowitz et al., 2000), the EMD subunit directional tuning function should indeed be narrower (Borst, 2004). Thus, identical computational mechanisms-in combination with the variations of the local spatial resolution of the compound eye-can explain the pattern motion response properties of both the $\mathrm{H} 1$ and V2 cells.

The tuning of the V1 cell was unimodal, and the peak preferred direction was not altered by the plaid separation angle (Fig.
$5 b$ ). The responses to $120^{\circ}$ and $150^{\circ}$ plaids were better fit by the unimodal predicted responses than the component cell model (Fig. $5 c$ ), resulting in a significantly higher correlation with the pattern cell predictions than the component cell predictions, for 5 of 6 cells (Fig. $5 c ; p<0.01 ; 120^{\circ}$ plaid: mean $Z_{\mathrm{P}}-$ mean $Z_{\mathrm{C}}=$ 3.0, $150^{\circ}$ plaid: mean $Z_{\mathrm{P}}-$ mean $Z_{\mathrm{C}}=2.9, N=6$ ). The pattern selectivity of the $\mathrm{V} 1$ cell responses to the $150^{\circ}$ plaid was present within the $50 \mathrm{~ms}$ after the stimulus onset for 3 of the six cells $(p<$ $0.10)$, and for 4 of the 6 cells by $100 \mathrm{~ms}\left(p<0.10\right.$; mean $Z_{\mathrm{C}}-$ mean $Z_{\mathrm{P}}=4.82, N=6$ ). Thus, we found no clear evidence that the pattern selectivity of the V1 cell takes longer to develop than the component selectivity of the $\mathrm{H} 1$ cell, as it does in primate MT/V5 cells (Smith et al., 2005).

The tuning of the V1 cell to plaids differed from the pattern cell model predictions in two ways. There were secondary peak responses to the $120^{\circ}$ and $150^{\circ}$ plaids at plaid directions of $165^{\circ}$ and $375^{\circ}$ (Fig. $5 b$, gray arrows), and the response to the $60^{\circ}$ plaid was greater than to the $30^{\circ}$ plaid at the preferred direction (Fig. $5 b ; p<0.03$, paired $t$ test, $N=6$ ). The pattern selectivity of V1 was maintained at a lower contrast level of $20 \%\left(150^{\circ}\right.$ plaid: mean $\left.Z_{\mathrm{C}}-Z_{\mathrm{P}}=-1.66, N=5\right)$. 


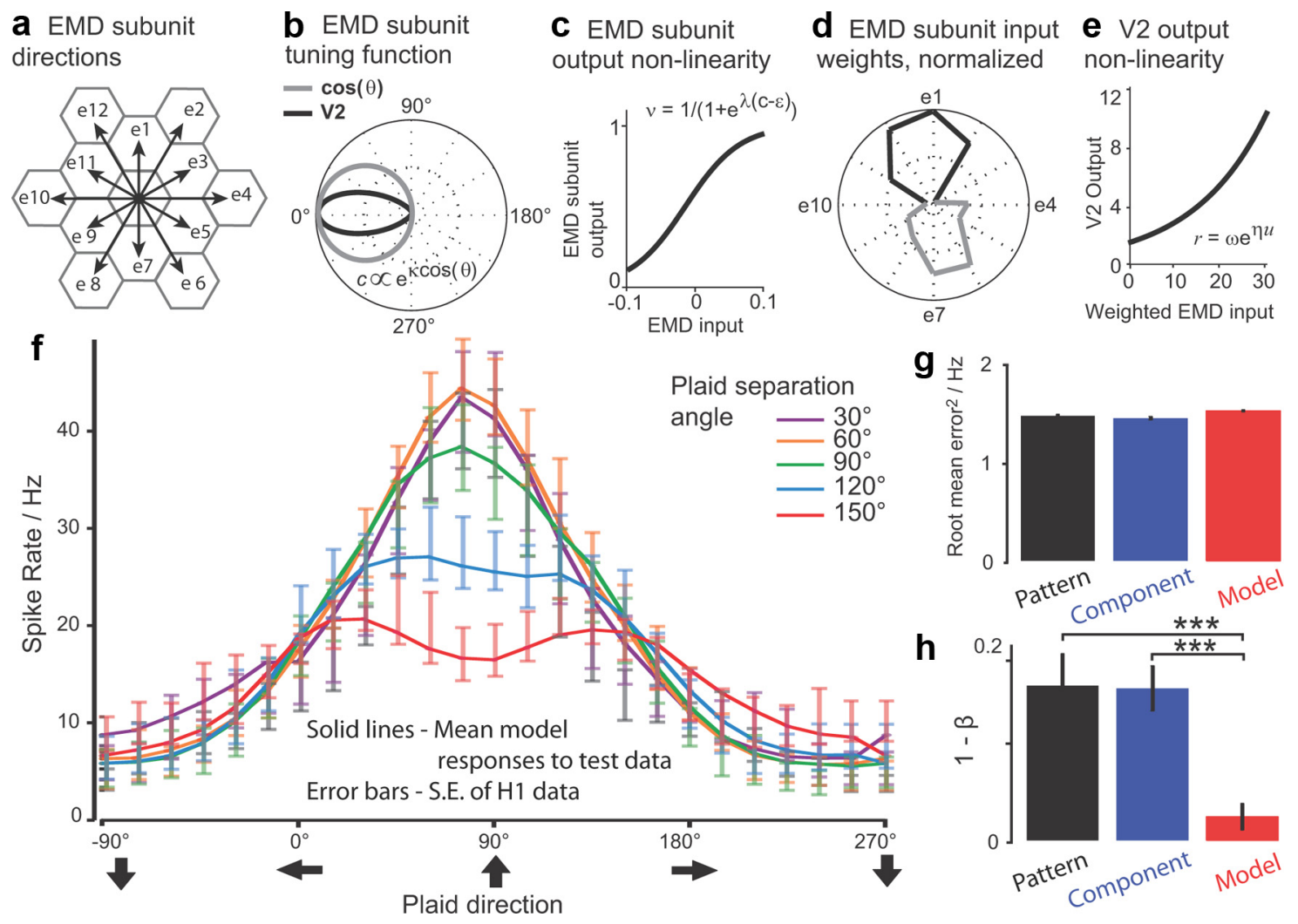

Figure 6. V2 cascade model. $\boldsymbol{a}-\boldsymbol{e}, \mathrm{V} 2$ cascade model parameters, fitted values and equations. a, The 12 directions of EMD subunits (black arrows). $\boldsymbol{b}$, EMD subunit directional tuning function. The fitted function (black) is shown with a cosine for comparison (gray). $\boldsymbol{c}$, EMD subunit output nonlinearity. $\boldsymbol{d}$, EMD subunit weight distribution. Excitatory (black) and inhibitory (gray) weights are shown. $\boldsymbol{e}, \mathrm{V} 2$ output nonlinearity. $\boldsymbol{f}$, Responses of V 2 cascade model to the test data (solid lines). Error bars show the variability of the V2 cell recordings, to demonstrate the accuracy of the V2 cascade model fit. $\boldsymbol{g}$, Root mean squared errors between V2 cascade model responses and predictions of the pattern and component models. $\boldsymbol{h}$, Fraction of explainable variance, $\beta$, accounted for by the models. $\boldsymbol{g}, \boldsymbol{h}$, Mean \pm SE shown $\left(N_{\text {fits }}=175\right)$.

\section{Discussion}

We have demonstrated that the blowfly's optomotor yaw response can be bimodally tuned to the direction of plaid stimuli, contrary to conclusions based on previous studies of twodimensional motion processing in flies (Fig. 1d). The directional tuning to plaids of the $\mathrm{H} 1$ cell contributing to this reflex was likewise bimodal for the $120^{\circ}$ and $150^{\circ}$ plaids (Fig. 2c). By fitting a cascade model to the recorded activity of the $\mathrm{H} 1$ cell (Fig. 3), we identified the essential features of neural processing required to capture the response of these cells to two-dimensional pattern motion: (1) a non-cosine direction tuning of EMD subunits (Fig. $4 a$ ), and (2) a saturating nonlinearity at the output of the EMD subunits (Fig. 4b). The directional tunings of the other LPTCs investigated here, the V1 and V2 cells, range from between pattern- and component-selective responses, to pattern-selective responses (Fig. 5), indicating that a component-like tuning is not a general property of the population of LPTCs.

We assumed that the LPTCs we recorded from were entirely driven by EMD inputs. A number of LPTCs have properties consistent with cells that process higher-order motion, such as the figure-detecting (FD) cells. FD cells respond to the motion of small objects in their receptive fields and are thought to support object fixation behavior (Egelhaaf, 1985a,b; Hausen and Wehrhahn, 1990). Flies produce yaw optomotor responses when challenged with second order motion which are not fully accounted for by integrated EMD signals alone (Theobald et al., 2008). While it is possible that non-EMD-based visual processing contributes to the bimodal directional tuning of the yaw optomotor reflex during plaid stimulation, EMD inputs provide the most parsimonious explanation of the inputs driving the $\mathrm{H} 1$ and V2 LPTCs (Quenzer and Zanker, 1991; Tuthill et al., 2011).

The behavioral responses to plaids have not been widely studied in invertebrate systems. Freely flying honeybees can learn to discriminate between the orientations of specific plaid patterns (Srinivasan et al., 1994). A flight situation that would generate plaid-like stimuli, for instance when the foreground and background appear to move in divergent directions, will likely generate additional visual motion cues, such as motion parallax or figure-ground discrimination, which the fly may use to guide its flight. For the behavioral responses driven by EMD inputs, our results and analysis show that neurons involved in the yaw response may allow the fly to preferentially follow one of the motion cues.

To our knowledge, the responses of motion-sensitive visual interneurons to plaids have not been investigated in detail in any invertebrate system. Eriksson $(1984,1989)$ recorded the responses of the H1 (Eriksson, 1984) and V1 (Eriksson, 1989) cells to two moving dots, when the direction of the second dot was varied. The responses of these cells were unchanged when the velocity of both dots along the preferred direction was held constant (Eriksson, 1984, 1989). These previous findings are consistent with our data for the V1 cell (Fig. 5b), but in apparent contradiction with our $\mathrm{H} 1$ cell data (Fig. 2). A possible explanation for the latter discrepancy between our results and the studies by Erikson may be that the velocity of the second dot varied with 
its motion direction. It is likely that changes in sensitivity to the direction of dot motion were affected by changes in the velocity.

The cascade model shows how the non-cosine direction tuning of EMD subunits generates a bimodal tuning to the plaids with large separation angles. A non-cosine directional tuning function is predicted by the EMD model (Varju, 1959; Götz, 1964; Zanker, 1990; Borst, 2004), but previous work indicated that it should only have an impact for much shorter spatial wavelengths than those used in our study (Zanker, 1990). For single grating stimuli of a spatial wavelength $>4$ times the inter-ommatidial angle $(\Delta \varphi)$ (Zanker, 1990), for both recordings and simulations of the $\mathrm{H} 1$ cell responses, the integration of multiple EMD outputs resulted in a directional tuning curve that was more similar to a sinusoid than the output of a modeled EMD. From this it was concluded that the noncosine direction tuning of EMDs has little impact on the directional responses of LPTCs and the animal's optomotor behavior, with the possible exception of cases when wavelengths $<4 \Delta \varphi$ are used. All previous studies have assumed, therefore, that the response to 2-dimensional patterns of visual motion is well approximated by the dot product between the direction of stimulus motion and the cell's preferred direction (Reichardt et al., 1988; Reichardt and Schlögl, 1988; van Hateren, 1990; Borst and Egelhaaf, 1993; Borst et al., 1993; Franz and Krapp, 2000; Weber et al., 2010). We have shown that the non-cosine direction tuning of EMD subunits can have a substantial impact on the LPTC and optomotor responses to visual stimuli that are more complex than single gratings, even when spatial wavelengths $>4 \Delta \varphi$ are used.

Our data provides evidence that the V1 cell is pattern-selective. Unlike the $\mathrm{H} 1$ cell, the $\mathrm{V} 1$ cell receives its motion inputs from three other LPTCs, the VS1-3 cells, and not direct EMD inputs (Hausen, 1984; Krapp et al., 1998). The VS1 cell is sensitive to horizontal motion in addition to vertical motion (Krapp and Hengstenberg, 1996), which is the likely reason for the secondary peaks in the V1 cell response. We did not model the $\mathrm{V} 1$ cell response because the extra layer of cellular processing, compared with the $\mathrm{H} 1$ and V2 cells, requires a greater number of model parameters than we could fit with our data. In the future, intracellular recordings from VS cells will allow the mechanisms behind the V1 cell's unimodal tuning to plaid direction to be established, and confirm whether the cell is indeed selective for pattern motion.

Our cascade model of the $\mathrm{H} 1$ and V2 cells is directly comparable to a recent model of the responses to plaid stimuli of neurons in macaque monkey visual cortex area V5/MT (Rust et al., 2006). Both models share the same overall structure, the main difference being the use of a saturating nonlinearity in our model instead of directionally tuned and untuned normalization terms. Tuned inhibition is a well explored concept in striate cortex, which provides the early stage of visual processing in the MT model. Although tuned inhibition of the EMD subunits has not been described, lateral inhibition of some form has been suggested to operate in the early stages of visual processing in insects (Srinivasan et al., 1982; Zheng et al., 2006; Bolzon et al., 2009). By contrast, a saturating output nonlinearity has often been used in models of EMDs (Zanker, 1990; Franz and Krapp, 2000). In our $\mathrm{H} 1$ cascade model, the saturating output nonlinearity limits the maximum responses to motion in the preferred direction, achieving a comparable modulation of the cell's response as tuned inhibition achieves for the cascade model of V5/MT cells (Rust et al., 2006).

The responses of the LPTCs to plaid stimuli are qualitatively similar to those of neurons found in V5/MT, which may vary along the continuum between pattern and component selectivity (Movshon et al., 1985). The calculated directional tuning curves of the EMD subunits are much broader than those of V1 (striate cortex) neurons. As a result, the challenge here is to explain how LPTCs generate a bimodal tuning to the plaid direction from broadly tuned EMD subunit responses, whereas for V5/MT it is to explain how a pattern cell-like response can be generated in some MT neurons from narrowly tuned V1 (striate cortex) neurons. In the model proposed by Rust et al. (2006), a broad distribution of excitatory and inhibitory weights generate a pattern motion selective response from narrowly tuned V1 (striate cortex) inputs. Rust et al. (2006) implemented tuned inhibition to ensure MT cell peak responses to plaid stimuli exceeded those to a single grating. In our $\mathrm{H} 1$ and V2 cascade models, the noncosine directional tuning of the EMD subunits generated a bimodal tuning to the plaid direction, and the saturating EMD subunit output function ensured that the peak response to the $60^{\circ}$ plaid exceeded that of the $30^{\circ}$ plaid. Thus, our model shows how functionally comparable mechanisms may explain the MT and LPTC responses to plaid motion stimuli.

In summary, we have identified the processing steps necessary to explain an unexpected behavioral response to two-dimensional patterns of motion in the blowfly. The results also indicate that the fly may employ a range of visual processing to support behavioral responses to multiple motion cues. By taking advantage of the fly's well characterized visuomotor pathway, future recordings from identified cells will allow us to further specify the underlying physiological mechanisms.

\section{References}

Adelson EH, Bergen JR (1985) Spatiotemporal energy models for the perception of motion. J Opt Soc Am A 2:284-299.

Adelson EH, Movshon JA (1982) Phenomenal coherence of moving visual patterns. Nature 300:523-525.

Bialek W, Rieke F, de Ruyter van Steveninck RR, Warland D (1991) Reading a neural code. Science 252:1854-1857.

Bolzon DM, Nordström K, O'Carroll DC (2009) Local and large-range inhibition in feature detection. J Neurosci 29:14143-14150.

Borst A (2004) Modelling fly motion vision. In: Computational neuroscience: a comprehensive approach (Feng J, ed), pp 397-429. Boca Raton: Chapman and Hall/CTC.

Borst A, Egelhaaf M (1993) Detecting visual motion: theory and models. In: Visual motion and its role in the stabilization of gaze, Vol 3 (Miles FA, Wallman J, eds), pp 3-27. Amsterdam: Elsevier.

Borst A, Haag J (2002) Neural networks in the cockpit of the fly. J Comp Physiol A Neuroethol Sens Neural Behav Physiol 188:419-437.

Borst A, Egelhaaf M, Seung HS (1993) Two-dimensional motion perception in flies. Neural Comput 5:856-868.

Brainard DH (1997) The psychophysics toolbox. Spat Vis 10:433-436.

Buchner E (1976) Elementary movement detectors in an insect visual system. Biol Cybern 24:85-101.

Buchner E (1984) Behavioural analysis of spatial vision in insects. In: Photoreception and vision in invertebrates (Ali MA, ed), pp 561-621. New York: Plenum.

Eckert H (1980) Functional properties of the H1-neurone in the third optic ganglion of the blowfly, Phaenicia. J Comp Physiol A Neuroethol Sens Neural Behav Physiol 135:29-39.

Egelhaaf M (1985a) On the neuronal basis of figure-ground discrimination by relative motion in the visual-system of the fly. 2. Figure-detection cells, a new class of visual interneurones. Biol Cybern 52:195-209.

Egelhaaf M (1985b) On the neuronal basis of figure-ground discrimination by relative motion in the visual-system of the fly. 3. Possible input circuitries and behavioral significance of the FD-cells. Biol Cybern 52:267-280.

Egelhaaf M, Borst A (1993) Movement detection in arthropods. In: Visual motion and its role in the stabilization of gaze (Miles FA, Wallman J, eds), pp 53-77. Amsterdam: Elsevier.

Eriksson ES (1984) Vector analysis in a neural network. J Insect Physiol 30:363-368. 
Eriksson ES (1989) Neural vector analysis and ambiguity in motion sensitive neurones in the brain of the blowfly. Physica Scripta 39:141-146.

Franz MO, Krapp HG (2000) Wide-field, motion-sensitive neurons and matched filters for optic flow fields. Biol Cybern 83:185-197.

Gabbiani F, Krapp HG, Koch C, Laurent G (2002) Multiplicative computation in a visual neuron sensitive to looming. Nature 420:320-324.

Götz KG (1964) Optomotorische Untersuchungen des visuellen systems einiger Augenmutanten der Fruchtfliege Drosophila. Kybernetik 2:77-92.

Haag J, Borst A (2001) Recurrent network interactions underlying flowfield selectivity of visual interneurons. J Neurosci 21:5685-5692.

Harris RA, O'Carroll DC, Laughlin SB (2000) Contrast gain reduction in fly motion adaptation. Neuron 28:595-606

Hartline HK, Wagner HG, Ratliff F (1956) Inhibition in the eye of Limulus. J Gen Physiol 39:651-673.

Hassenstein B, Reichardt W (1956) Systemtheoretische Analyse der Zeit-, Reihenfolgen- und Vorzeichenauswertung bei der Bewegungsperzeption des Rüsselkafers Chlorophanus. Z Naturforsch 11:523-524.

Hastie T, Tibshirani R, Friedman J (2001) The elements of statistical learning: data mining, inference and prediction. New York: Springer.

Hausen K (1984) The lobular complex of the fly: structure, function and significance in visual behaviour. In: Photoreception and vision in invertebrates (Ali MA, ed), pp 523-559. New York: Plenum.

Hausen K (1993) The decoding of retinal image flow in insects. In: Visual motion and its role in the stabilization of gaze (Miles FA, Wallman J, eds), pp 203-235. Amsterdam: Elsevier.

Hausen K, Wehrhahn C (1983) Microsurgical lesion of horizontal cells changes optomotor yaw responses in the blowfly Calliphora erythrocephala. Proc R Soc Lond B Biol Sci 219:211-216.

Hausen K, Wehrhahn C (1989) Neural circuits mediating visual flight control in flies. I. Quantitative comparison of neural and behavioral response characteristics. J Neurosci 9:3828-3836.

Hausen K, Wehrhahn C (1990) Neural circuits mediating visual flight control in flies. II. Separation of two control systems by microsurgical brain lesions. J Neurosci 10:351-360.

Krapp HG, Hengstenberg R (1996) Estimation of self-motion by optic flow processing in single visual interneurons. Nature 384:463-466.

Krapp HG, Wicklein M (2008) Central processing of visual information in insects. In: The senses: a comprehensive reference (Bushnell MC, Smith DV, Beauchamp GK, Firestein SJ, Dallos P, Oertel D, Masland RH, Albright T, Kaas JH, Gardner EP, Basbaum AI, eds), pp 131-204 San Diego: Academic.

Krapp HG, Hengstenberg B, Hengstenberg R (1998) Dendritic structure and receptive-field organization of optic flow processing interneurons in the fly. J Neurophysiol 79:1902-1917.

Krapp HG, Hengstenberg R, Egelhaaf M (2001) Binocular contributions to optic flow processing in the fly visual system. J Neurophysiol 85:724734.

Longden KD, Krapp HG (2009) State-dependent performance of optic-flow processing interneurons. J Neurophysiol 102:3606-3618.

Machens CK, Wehr MS, Zador AM (2004) Linearity of cortical receptive fields measured with natural sounds. J Neurosci 24:1089-1100.

Movshon JA, Albright TD, Gizzi MS, Newsome WT (1985) The analysis of visual moving patterns. In: Pattern recognition mechanisms (Chagas C, ed), pp 148-164. New York: Springer.

Movshon JA, Albright TD, Stoner GR, Majaj NJ, Smith MA (2003) Cortical responses to visual motion in alert and anesthetized monkeys. Nat Neurosci 6:3.

Nelder JA, Mead R (1965) A simplex method for function minimization. Comput J 7:308-313.

Newsome WT, Britten KH, Movshon JA (1989) Neuronal correlates of a perceptual decision. Nature 341:52-54.

Petrowitz R, Dahmen H, Egelhaaf M, Krapp HG (2000) Arrangement of optical axes and spatial resolution in the compound eye of the female blowfly Calliphora. J Comp Physiol A Neuroethol Sens Neural Behav Physiol 186:737-746.

Quenzer T, Zanker JM (1991) Visual detection of paradoxical motion in flies. J Comp Physiol A Neuroethol Sens Neural Behav Physiol 169:331-340.

Reichardt WE, Schlögl RW (1988) A two dimensional field theory for motion computation. First order approximation; translatory motion of rigid patterns. Biol Cybern 60:23-35.

Reichardt W, Egelhaaf M, Schlögl RW (1988) Movement detectors provide sufficient information for local computation of 2-D velocity field. Naturwissenschaften 75:313-315.

Rust NC, Mante V, Simoncelli EP, Movshon JA (2006) How MT cells analyze the motion of visual patterns. Nat Neurosci 9:1421-1431.

Sanes JR, Zipursky SL (2010) Design principles of insect and vertebrate visual systems. Neuron 66:15-36.

Simoncelli EP, Heeger DJ (1998) A model of neuronal responses in visual area MT. Vision Res 38:743-761.

Smith MA, Majaj NJ, Movshon JA (2005) Dynamics of motion signaling by neurons in macaque area MT. Nat Neurosci 8:220-228.

Srinivasan MV, Laughlin SB, Dubs A (1982) Predictive coding: a fresh view of inhibition in the retina. Proc R Soc Lond B Biol Sci 216:427-459.

Srinivasan MV, Zhang SW, Witney K (1994) Visual discrimination of pattern orientation by honeybees: performance and implications for cortical processing. Philos Trans R Soc B 343:199-210.

Theobald JC, Duistermars BJ, Ringach DL, Frye MA (2008) Flies see second-order motion. Curr Biol 18:R464-R465.

Tuthill JC, Chiappe ME, Reiser MB (2011) Neural correlates of illusory motion perception in Drosophila. Proc Natl Acad Sci U S A 108:9685-9690.

van Hateren JH (1990) Directional tuning curves, elementary movement detectors, and the estimation of the direction of visual movement. Vision Res 30:603-614.

Varju D (1959) Optomotorische Reaktionen Auf Die Bewegung Periodischer Helligkeitsmuster - (Anwendung Der Systemtheorie Auf Experimente Am Russelkafer Chlorophanus viridis). Z Naturforsch Pt B 14:724-735.

Weber F, Machens CK, Borst A (2010) Spatiotemporal response properties of optic-flow processing neurons. Neuron 67:629-642.

Zanker JM (1990) On the direction sensitivity of motion detectors. Biol Cybern 62:177-182.

Zheng L, de Polavieja GG, Wolfram V, Asyali MH, Hardie RC, Juusola M (2006) Feedback network controls photoreceptor output at the layer of first visual synapses in Drosophila. J Gen Physiol 127:495-510. 